Extrême-Orient Extrême-Occident

\section{Extrême-Orient Extrême-Occident}

$36 \mid 2013$

Mobilité humaine et circulation des savoirs techniques (XVII ${ }^{\mathrm{e}} \mathrm{XIX}$ siècles)

\title{
Les traductions de François-Xavier Dentrecolles (1664-1741), missionnaire en Chine : localisation et circulation des savoirs
}

The Translations of François-Xavier Dentrecolles (1664-1741), Missionary in China: Locality and the Circulation of Knowledge

在華傳教士殷弘緒（1664-1741）的翻譯活動：地域特徵與知識流動

\section{Huiyi Wu}

\section{(2) OpenEdition}

\section{Journals}

Édition électronique

URL : http://journals.openedition.org/extremeorient/306

DOI : $10.4000 /$ extremeorient.306

ISSN : 2108-7105

Éditeur

Presses universitaires de Vincennes

Édition imprimée

Date de publication : 31 décembre 2013

Pagination : 49-80

ISBN : 978-2-84292-404-1

ISSN : 0754-5010

Référence électronique

Huiyi Wu, « Les traductions de François-Xavier Dentrecolles (1664-1741), missionnaire en Chine : localisation et circulation des savoirs ", Extrême-Orient Extrême-Occident [En ligne], 36 | 2013, mis en ligne le 01 juin 2017, consulté le 30 avril 2019. URL : http://journals.openedition.org/ extremeorient/306; DOI : 10.4000/extremeorient.306 


\section{Les traductions de François-Xavier Dentrecolles (1664-1741), missionnaire en Chine : localisation et circulation des savoirs}

Wu Huiyi

L'une des images les plus tenaces du missionnaire jésuite en Chine est celle d'un homme de cour, mettant son talent d'astronome, d'artiste ou de médecin au service de l'empereur qu'il espère gagner à la religion chrétienne. Il est vrai que beaucoup de jésuites gardaient à l'esprit l'idée d'une évangélisation de la Chine par le haut, sur le modèle de la conversion de l'empereur Constantin à Rome, et que la cour et les institutions impériales ont été des lieux privilégiés d'échange de savoirs. Mais la sur-visibilité de Pékin ne doit pas faire oublier que la plupart des effectifs de la mission ont toujours été déployés dans les provinces. Un catalogue du personnel de la mission française montre qu'en 1710, parmi les trente jésuites dépendant de la mission française, six seulement sont à Pékin (carte 1) ${ }^{1}$. Les activités apostoliques des missionnaires de province font aujourd'hui l'objet de nombreux travaux de recherche; mais on sait encore relativement peu de choses sur leurs activités intellectuelles, notamment sous les Qing, alors que les «sciences jésuites» se recentrent sur la cour.

1. Archivum Romanum Societatis Iesu (ARSI), Japonica-Sinica (JS) 134, f ${ }^{\circ}$ 390-391. Le document ne comporte pas de date, mais on peut affirmer qu'il date de 1710 ou 1711 puisque Guillaume Melon (1666-1710) y est signalé comme «mort en 1710 », et que Jean-François Foucquet, qui part pour Pékin en 1711, y est encore localisé à Linjiang, au Jiangxi. 


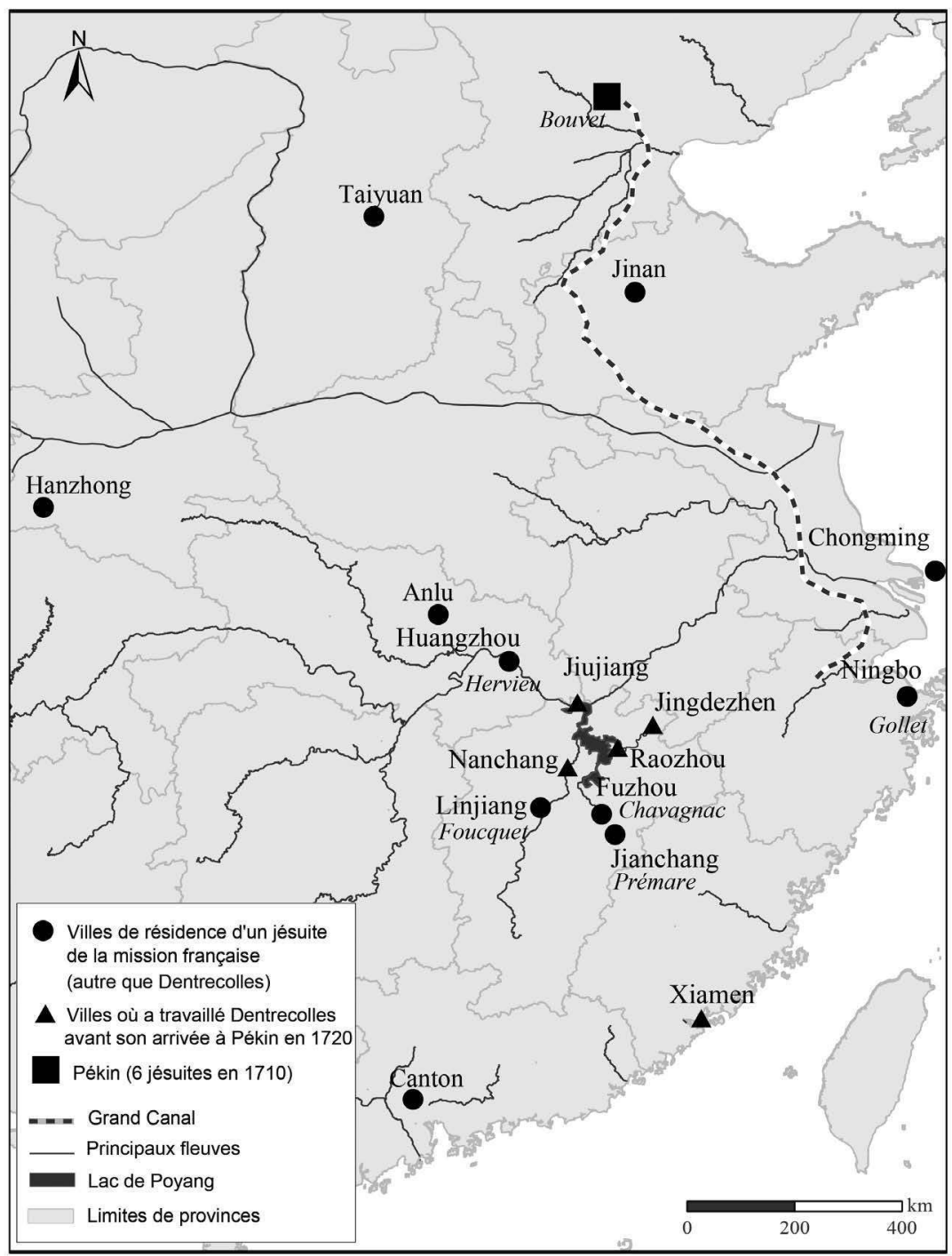

Source: ARSI, JS 134, 10390-391, Réalisation: Antoine Fivel, 2013

Carte 1. La mission jésuite française de Chine vers 1710 
Le rédacteur de ce catalogue, François-Xavier Dentrecolles (1664-1741) ${ }^{2}$, supérieur de la mission française de 1707 à 1719, est précisément l'un de ces jésuites qui n'ont jamais travaillé à la cour de Pékin. Auteur de contributions majeures aux deux publications qui ont profondément renouvelé la connaissance européenne de la Chine - les Lettres édifiantes et curieuses (Paris, 1702-1776, ci-après $(L E C)^{3}$ et la Description de l'Empire de la Chine et de la Tartarie chinoise (Paris, 1735, ci-après Description) ${ }^{4}$-, Dentrecolles a passé vingt ans dans les provinces, d'abord brièvement au Fujian (1699-ca fin 1700), puis au Jiangxi (ca 1701-1719). S'il passe la seconde moitié de sa carrière chinoise à Pékin (1720-1741), c'est d'abord en tant que supérieur de la résidence jésuite, sans jamais servir les empereurs ${ }^{5}$. Dentrecolles est surtout connu pour son enquête sur la technique chinoise de la porcelaine; mais il a aussi écrit sur un grand nombre d'autres sujets, depuis la variolisation et l'élevage des vers à soie jusqu'au système éducatif et à la littérature vernaculaire. L'invariant, par-delà cet éclectisme, c'est l'importance que Dentrecolles accorde aux sources livresques qu'il traduit du chinois. Nous possédons déjà une première synthèse sur sa carrière et son œuvre grâce à $\mathrm{M}^{\text {me }}$ Thomaz de Bossierre ${ }^{6}$; dans les pages qui suivent, nous nous proposons de jeter un éclairage nouveau sur les conditions de production de ces œuvres en les replaçant dans l'itinéraire de Dentrecolles esquissé ci-dessus. À travers cet exemple, il s'agit d'une part de mettre en question l'historiographie traditionnelle, qui tend à faire entrer dans l'histoire des savoirs les seuls jésuites de cour tandis que leurs confrères des provinces sont décrits comme se consacrant presque exclusivement à l'évangélisation, et d'autre part de mettre en lumière la pertinence et les limites de l'hypothèse d'une corrélation forte entre les savoirs et leurs lieux de production.

2. L'identification de Dentrecolles comme auteur de ce catalogue est faite sur une base graphologique. De plus, il est le seul dont les compétences n'y sont pas évaluées.

3. J'utilise l'édition d'Aimé-Martin [1702-1776] 1853.

4. Du Halde 1735: tome 1, lj-lij; Landry-Deron 2002 : 68-70.

5. Gaubil au P. Gaillard, Pékin, le 8 oct. 1727, «Les PP. Derezende et Dentrecolles ne sont pas au service de l'empr. Le feu empr. les souffroit icy, on ne leur a pas dit de s'en aller, et jusqu'ici on n'a craint pour eux aucune avanie.» (Gaubil 1970 : 181.)

6. Thomaz de Bossierre 1982. 


\section{Province de Lyon (1682-1698)}

Le nom de Dentrecolles commence à apparaître dans les sources historiques lors de son entrée dans la Compagnie de Jésus : nous savons par les catalogues du personnel - outil administratif fournissant une synthèse succincte des «moyens humains » de chaque collège jésuite - que François-Xavier Dentrecolles est né en 1664 à Limoges, qu'il est entré au noviciat en 1682 à Avignon, et qu'il est ensuite passé par les collèges de Lyon et de Pignerol ${ }^{7}$. Son parcours européen se cantonne donc à la province de Lyon $^{8}$ : à la différence des jésuites italiens ou allemands qui passaient tous par le Portugal pour se rendre en Chine, il ne semble jamais avoir étudié dans un autre pays que la France et n'a sans doute jamais vu Paris.

Le catalogue de 1696 nous apprend que ses supérieurs le jugent apte «à prêcher et à gouverner», qu'il a enseigné six ans en classe de grammaire et deux ans en classe de rhétorique; son caractère y est décrit, selon la typologie de la médecine humorale, comme «sanguin à tendance mélancolique ${ }^{9} »$. D'après ces maigres indications administratives, souvent réduites à un simple «bonus » - accordé à un assez grand nombre ${ }^{10}$, rien ne le distingue du commun des membres les plus jeunes de la Compagnie. Il n'a rien publié avant son départ pour la Chine ${ }^{11}$, et ne semble pas avoir fait preuve de talent «scientifique» aux yeux de sa hiérarchie: ses huit années d'enseignement ne comprennent pas d'enseignement de mathématiques ou d'humanités (qui incluent la philosophie naturelle). Le seul éclairage personnel sur sa période européenne provient d'une lettre de 1713, dans laquelle il rend compte de sa vocation pour la Chine - apparemment dépourvue d'ambition savante - en citant pour modèle le jésuite missionnaire de campagne Jean-François Régis (1597-1640) ${ }^{12}$ :

7. Son nom apparaît dans le catalogue des collèges: celui d'Avignon jusqu'en 1690 (ARSI, Lugd [unensis] 22, f ${ }^{\circ}$ 361), celui de Lyon en 1693 (Ludg. 23, fo 5), celui de Pignerol (actuel Pinerolo en Italie) en 1696 (Lugd. 23, $\mathrm{f}^{\circ}$ 167).

8. La Compagnie de Jésus compte alors cinq «provinces» dans le Royaume de France: «France» (qui recouvre à cette époque la partie nord-ouest du royaume, y compris la Bretagne, la Normandie et Paris), Lyon, Champagne, Aquitaine, Toulouse.

9. $\quad$ ARSI, Lugd. 23, f $\mathrm{f}^{\circ} 210$.

10. En 1690, par exemple, dans la colonne «profectus in litteris », Dentrecolles est jugé «bonus in omnibus»

11. Sommervogel [1941] 1960 : vol. 2, 1932-1935, «d'Entrecolles».

12. Jean-François Régis a travaillé comme «missionnaire de l'intérieur» dans les monts du Vivarais et dans les Cévennes. Il meurt à Lalouvesc (Ardèche) qui devient vite un lieu de pèlerinage. En 1737, il est le premier jésuite français à être canonisé. Sur la vie de Régis, voir Ortroy 1910. 
Les traductions de François-Xavier Dentrecolles, missionnaire en Chine

Et afin que V[otre] R[évéren] ce reussisse pour la canonization du vénérable $\mathrm{P}$. Regis, qu'elle a si a cœur, ie dirai plusieurs messes [...] à son intention; i'ai esté plusieurs fois à son tombeau et ie crois luy devoir mon entrée à la Compagnie, ma vocation à la Chine, et l'execution de cette vocation de maniere que ie ne me suis pas donné aux portugais, comme ie la [?] resolu d'aller faire mes estudes a Goa, lorsque i'allai faire un pelerinage a La Louvesc pour consulter mon bon sainct à la fin de ma regence, ce qui empecha que ie ne demande a passer en Portugal ${ }^{13} \ldots$

\section{La mer et le Fujian, antichambre de la Chine (février 1698-fin 1700)}

Dentrecolles fait partie de la dizaine de missionnaires recrutés entre 1697 et 1698 par l'un des membres fondateurs de la mission jésuite française en Chine, Joachim Bouvet (1656-1730). Ce dernier était alors de retour en France en qualité d'envoyé de l'empereur Kangxi, au service duquel il se trouvait depuis son arrivée en Chine en $1688^{14}$. Mais Le début de la carrière missionnaire de Dentrecolles, que l'on peut reconstituer à partir de sources fragmentaires, ne ressemble guère au récit conventionnel du parcours des jésuites en Chine. Bouvet avait gardé la majorité des nouvelles recrues auprès de lui à bord de l'Amphitrite qui va «en droiture» de La Rochelle à Canton ${ }^{15}$. Il leur donne des cours de chinois et de mandchou pendant le voyage ${ }^{16}$, et le groupe qui l'accompagne est accueilli en grande pompe par les fonctionnaires locaux, grâce au statut d'envoyé impérial de Bouvet ${ }^{17}$. Cependant, Dentrecolles et deux de ses confrères, Jean-François Foucquet (1665-1741) et Pierre Frapperie (1664-1703), ne font pas le voyage avec Bouvet mais avec l'escadre d'un certain Chevalier des Augers qui avait pour destination Chandernagor au Bengale. Multipliant les escales, passant par Madras (où ils sont rejoints par Jean de Pélisson [1657-1713] et Bernard Rhodes [1646-1715]), Batavia (actuel Jakarta), Palembang (au sud de Sumatra) et Malacca, à bord de divers navires marchands anglais, ce groupe finit par atteindre Xiamen (connu dans les

13. Dentrecolles à Daubenton, Jiujiang, le 23 juin 1713, ARSI, JS 174, f 493.

14. Sur les débuts de la mission française en Chine avec l'envoi de cinq jésuites connus sous le nom de «Mathématiciens du Roi», voir Landry-Deron 2001 : 423-463. Pour la biographie de Joachim Bouvet, voir von Collani 1985.

15. L'Amphitrite est le premier navire marchand français à voguer directement vers les côtes chinoises (Pelliot 1930).

16. Ce détail nous est appris par le journal de navigation de François Froger, l'un des seconds de l'Amphitrite lors de ce premier voyage: «Le 15, 16, 17 et 18, nous eumes les vens variables du Sud Sudoüest au Nordouëst, bon frais, beau tems. Le Pere Bouuet commença ses leçons pour les langues Chinoise et Tartare.» (Froger 1926 : 3.)

17. Bouvet à de la Chaize, le 30 novembre 1699. (LEC: 17-22.) 


\section{Wu Huiyi}

sources de l'époque sous le nom d'Emoui ou d'Amoy) le 24 juillet 1699, après un voyage de dix-sept mois, neuf mois de plus que celui de leurs compagnons arrivés à bord de l'Amphitrite ${ }^{18}$.

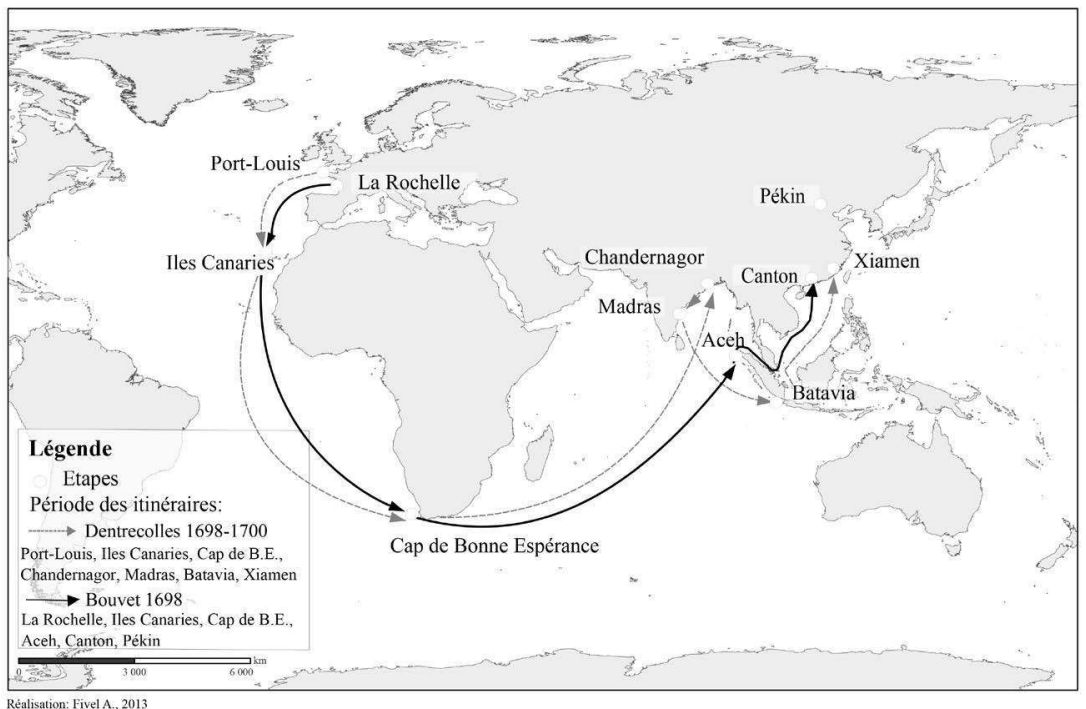

Carte 2. Deux itinéraires des jésuites français vers la Chine (1698-1700)

À leur arrivée, ils ne reçoivent aucun accueil des autorités chinoises. Les nouveaux arrivants sont pris en charge par le réseau missionnaire local, et sont par conséquent immédiatement plongés dans les conflits internes à ce réseau. L'histoire de la mission catholique en Chine, en ce tournant du XVIII ${ }^{\mathrm{e}}$ siècle, est marquée par la «querelle des rites chinois»: la politique d' «accommodation» que les jésuites appliquent depuis Matteo Ricci (1552-1610) est remise en cause par les missionnaires des autres ordres, qui trouvent qu'elle implique trop de compromis avec les traditions locales ${ }^{19}$. La situation est particulièrement complexe au Fujian, une province côtière assez fortement christianisée mais

18. Dentrecolles n'ayant pas laissé de récit de voyage, c'est grâce au journal de Foucquet, son unique compagnon d'un bout à l'autre, que nous connaissons les détails du voyage. Ce journal est conservé à la Bibliothèque apostolique vaticane (Borgia Latino 523) et a été étudié par Witek $1982: 87-92$.

19. Standaert (éd.) $2001: 680-682$. 
où les différents ordres présents - jésuites, dominicains, Missions étrangères de Paris (MEP) - s'opposent sur de la question des rites ${ }^{20}$. Or, dans un premier temps, c'est Charles Maigrot, MEP (1652-1730), qui offre l'hospitalité à ce petit groupe de jésuites français. Vicaire apostolique du Fujian, Maigrot a joué un rôle clé dans l'intensification de la querelle des rites en émettant en 1693 un fameux mandement (le «mandement de Maigrot») interdisant aux chrétiens chinois de pratiquer certains rites, notamment ceux liés au culte des ancêtres; cette mesure prohibitive allait à l'encontre de la pratique des jésuites. Un fragment d'une lettre de Dentrecolles écrite deux mois après son arrivée, retranscrit par une main anonyme et conservé à la Bibliothèque nationale de France $(\mathrm{BnF})$ dans un volume intitulé Anecdotes Orientales, nous apprend pourtant que son séjour à la résidence des MEP à Xiamen s'est fort bien passé : Dentrecolles fait l'éloge de son hôte et exprime sa gratitude envers lui avec un «mot chinois», ce qui suggère qu'il s'est initié aux premiers éléments de la langue au cours de cette période ${ }^{21}$. Foucquet, de son côté, a préféré quitter Xiamen dès le début de mois d'août pour rejoindre le jésuite italien Gianpaolo Gozani (1659-1732) à Fuzhou (Fujian) ${ }^{22}$. Ces choix divergents illustrent l'ambiguïté du petit monde missionnaire, dans lequel la collaboration est souvent une nécessité, et l'opposition entre différentes factions, bien que réelle, peut s'avérer toute relative.

Cette opposition va pourtant soudain éclater au grand jour en 1700. À la veille de Pâques, Maigrot, qui vient d'être consacré évêque, décide d'imposer l'interdiction des rites chinois à Gozani, sous peine de suspension de son ministère. Lorsque la nouvelle se répand chez les chrétiens de Fuzhou (Fujian), qui se trouvent sous l'autorité du jésuite, l'opinion s'enflamme. Le 18 avril, un rassemblement devant l'église de Maigrot dégénère en bagarre, au cours de laquelle des armes auraient été utilisées, si l'on en croit le compte rendu des MEP. Celui-ci porte un titre dramatique et accusateur: "Lettre écrite de la province de Fukien dans la Chine sur la fin de l'année 1700 où l'on rapporte le cruel traitement que les chrétiens des Jésuites ont fait à Mr. Maigrot, Évêque de Conon \& Vicaire Apostolique, au R. P. Croquet de l'ordre de S. Dominique; $\&$ où l'on voit un échantillon du respect qu'ont les Jésuites pour les Évêques \& pour S. Siège $e^{23}$.»

Dentrecolles n'a laissé aucun commentaire sur cet incident qui survient après qu'il a passé à peine neuf mois dans le pays : les missionnaires s'entredéchirent,

20. Menegon $2009: 59-116$.

21. Bibliothèque nationale de France $(\mathrm{BnF}), \mathrm{Ms}$ Fr. 25056, $\mathrm{f}^{\circ} 83$.

22. Witek $1982: 94-102$.

23. Wu Min et Han Qi 2004 : 83-91. 
des chrétiens se révoltent au nom de leurs traditions contre un évêque qu'il a côtoyé et auquel il est reconnaissant de son hospitalité. D'après les Anecdotes Orientales, en octobre 1700, Dentrecolles est toujours à Xiamen, avec deux prêtres des MEP et un dominicain. Très démoralisé, il fait part de sa désillusion à un ancien collègue de Lyon, auquel il confie qu'il «estime bien heureux le P. Burin d'avoir été appelé par le Seigneur avant que d'arriver ici». Xiamen et ses environs où il a passé l'année entière sont décrits comme un champ stérile où il n'y a que « 3 chrétiens en tout», par opposition à «une province» où il se rend enfin, espérant y trouver «beaucoup de chrétiens ${ }^{24}$ ».

\section{Le Jiangxi (ca 1701-1719)}

Cette province est le Jiangxi, où Dentrecolles passera les vingt années suivantes de sa vie. La situation y est bien différente de celle du Fujian limitrophe: la présence missionnaire est alors relativement faible, laissant suffisamment de terrain vierge aux nouveaux arrivants: un bon nombre de jésuites français s'y installent en ce début des années $1700^{25}$. Malgré sa situation à l'intérieur des terres, le Jiangxi n'est pas isolé du monde. Au contraire, jusqu'au milieu du XIX ${ }^{\mathrm{e}}$ siècle, c'est un carrefour où se croisent deux voies de transport majeures: le fleuve Yangzi qui traverse l'empire d'ouest en est, et la route qu'empruntent toutes les ambassades étrangères allant de Canton à Pékin, en passant par Shaoguan, le Fleuve Gan, le Lac Poyang, le Yangzi et le Grand Canal. La douane de Jiujiang qui domine la jonction des deux voies enregistre alors le plus gros montant de taxes sur les marchandises de tout l'empire ${ }^{26}$. C'est également une région aux traditions lettrées fortes: outre les nombreux philosophes néo-confucéens originaires de cette province, y vivant ou y ayant vécu ${ }^{27}$, le Jiangxi a fourni sous la dynastie Ming (1368-1644) l'un des contingents les plus importants de lauréats au concours métropolitain de recrutement de fonctionnaires (jinshi $)^{28}$. Une étude approfondie de cette province - s'inspirant de la riche historiographie existant sur le Jiangnan voisin - serait certainement bienvenue; contentons-nous ici

24. BnF, Ms Fr. 25056, $\mathrm{f}^{\circ} 83$.

25. Standaert 2012: 189-191.

26. Naquin et Rawski $1989: 162$.

27. L'historiographe des écoles néo-confucéennes Huang Zongxi (1610-1695) consacre neuf des soixante-deux juan (fascicules) du Ming ru xue an [Études des écoles confucéennes des Ming, 1676] aux «partisans de Wang Yangming au Jiangxi» (Jiangyou Wang men, Jiangyou, «rive droite du Fleuve», étant une dénomination du Jiangxi). (Huang Zongxi [1676] 1985, vol. 1 : 335-881.)

28. Ho Ping-ti 1964 : 226-231. 
de remarquer que pendant les deux premiers siècles de la dynastie Qing (pour être précise, du règne de Kangxi à celui de Daoguang, soit de 1662 à 1850), il y fut compilé le plus grand nombre de monographies locales ${ }^{29}$ : il n'est sans doute pas fortuit que le levé cartographique réalisé sur l'ordre de l'empereur Kangxi avec le concours des jésuites montre à l'endroit de cette province la plus grande précision : les monographies locales étaient en effet à la disposition des équipes chargées du levé ${ }^{30}$.

Après son arrivée au Jiangxi, les sources existantes sur Dentrecolles - récits publiés et correspondance avec sa hiérarchie - nous ramènent à une narration missionnaire plus conventionnelle. Son énorme correspondance traite presque exclusivement des progrès de la mission. L'image de lui qui s'en dégage est celle d'un missionnaire zélé, tout occupé à propager l'évangile parmi les classes populaires. Ses résultats doivent être suffisamment remarqués par ses supérieurs de Pékin et de Rome pour qu'il signe déjà la relatio missionis adressée au supérieur général en 1704, puis en $1706^{31}$, avant d'être nommé supérieur de la mission française en 1707, succédant ainsi à Jean-François Gerbillon (1654-1707) ${ }^{32}$.

À en juger par les lieux d'envoi de ses lettres, les vingt années que Dentrecolles a passées au Jiangxi représentent une période de mobilité constante: son siège principal se situe à Raozhou: il se rend régulièrement dans les villes de Nanchang, Jiujiang et Jingdezhen, reliées entre elles par des voies navigables (voir carte 1). C'est au cours de cette période, en 1712, qu'il rédige sa célèbre lettre sur la porcelaine de Jingdezhen - un savoir technique localisé sur le territoire où il exerce son ministère, et qui constitue un enjeu économique important pour la France. Au fil de son enquête, il «parcour [t] les boutiques des ouvriers, s'instrui $[\mathrm{t}$ ] de [s] es propres yeux » et pose «diverses questions aux chrétiens qui sont occupés à ce travail ${ }^{33} »$; il consulte au moins une source livresque, la Monographie locale du district de Fuliang (Fuliang xianzhi):

29. Naquin \& Rawski 1989: 165.

30. Wang 1995 : 143 évalue la précision de la carte de Kangxi province par province, en la comparant aux données obtenues par les techniques cartographiques modernes. Sur la mise des monographies locales à la disposition des cartographes, voir Cams 2012: 15.

31. ARSI, JS 168, fo 31-32 (1704), JS 169, f 378 (1706).

32. Sur Gerbillon, voir Thomaz de Bossierre 1994.

33. Dentrecolles au Père ***, le 25 janvier 1722. (LEC: 309.) 
Parmi ces livres, j'ai eu entre les mains l'histoire ou les annales de Feou-leam, et j'ai lu avec soin dans le quatrième tome l'article qui regarde la porcelaine ${ }^{34}$.

Les travaux de Dentrecolles durant cette période se comprennent mieux en mettant en lumière ce qu'il ne fait pas. Sa correspondance ne contient nul écho du riche héritage lettré de la région du Jiangxi, et son nom est remarquablement absent des activités collectives des jésuites français en matière d'interprétation de la philosophie chinoise. Car ces deux premières décennies du XviII ${ }^{\mathrm{e}}$ siècle marquent l'apogée du «figurisme», dont les tenants s'organisent autour de Bouvet. S'inscrivant dans le sillage de la politique d'accommodation lancée par Ricci, Bouvet va beaucoup plus loin, en défendant non seulement la compatibilité entre le christianisme et le confucianisme mais leur identité, et en considérant les canons chinois antiques, notamment le Yijing (Classique des Mutations), comme des livres révélés. Le figurisme de Bouvet peut être compris comme le fruit de la rencontre entre deux traditions numérologiques, celle de l'Europe et celle de la Chine, toutes deux encore fort dynamiques à son époque ${ }^{35}$. Mais il est aussi ancré dans le contexte plus particulier de la cour impériale, puisqu'il répond à l'intérêt de Kangxi pour le Yijing, s'adressant à l'empereur et à son entourage: c'est la raison pour laquelle Bouvet rédige ses ouvrages en chinois. L'un de ceux-ci, le Tian xue ben yi (Sens originel des études célestes), est présenté au légat du pape, Charles-Thomas Maillard de Tournon (1668-1710), à l'occasion de son arrivée à Canton le 8 avril 1705, comme l'un des éléments de la plaidoirie jésuite en faveur des rites chinois. Un premier rejet par le légat, qui n'est pas sinisant, oblige les jésuites à entreprendre une traduction latine de ce texte ${ }^{36}$. Ce travail est confié à un groupe de jésuites œuvrant au Jiangxi, tous issus du Collège de Paris. Une lettre qu'Alexis de Gollet (1764-1741) adresse depuis Ningbo à Gerbillon le 23 septembre 1705 révèle la composition et le fonctionnement de ce groupe:

$\mathrm{V}^{\mathrm{e}} \mathrm{R}^{\mathrm{ce}}$ a tres bien fait d'ordonner aux PP. Foucquet, de Premare (1666-1736), Hervieu (1671-1746) et Chavagnac (1670-1717) de s'assembler à Voutcheou (Fuzhou, Jiangxi) pour conférer ensemble et refuter les accusations qu'ils trouveront fausses dans les escrits de nos adversaires. Conformément a l'ordre de $\mathrm{V}^{\mathrm{e}} \mathrm{R}^{\mathrm{ce}}$, je vais aussi ecrire au cher P. Contancin (1671-1733) qu'il vienne me trouver icy pour y passer

34. Dentrecolles à Orry, Raozhou, le $1^{\text {er }}$ septembre 1712. (LEC : 207.)

35. Sur le figurisme de Bouvet, voir Mungello 307-328; von Collani 2007 : 227-387.

36. Sous le titre de Caelestis disciplinae vera notitia, Pistoia, Biblioteca Fabroniana, ms. 53. (Standaert (éd.) $2001: 676$. 
15 jours. J'y vay apres en passer autant chez luy pour travailler conjointement a l'abrégé latin de l'Apologie du P. Bouvet ${ }^{37}$.

Dentrecolles reste à l'écart de l'entreprise qui se déroule dans la province même où il réside. Il passe bientôt au rôle de censeur intransigeant, une fois devenu supérieur de la mission française. Entre avril et novembre 1711, lorsque Foucquet, son ancien compagnon de voyage devenu le plus fervent des figuristes, est convoqué à Pékin sur ordre impérial pour seconder Bouvet dans ses recherches sur le Yijing, Dentrecolles leur envoie une série de lettres, dont des copies sont conservées dans les archives de la Compagnie de Jésus à Rome, leur enjoignant d'être prudents ${ }^{38}$. Ses arguments sont d'abord politiques : il ne faut pas donner de grain à moudre aux détracteurs des jésuites au sein de l'Église, en prétendant que le Yijing permet de calculer le terme de la fin du monde ${ }^{39}$; il ne faut pas non plus risquer de paraître défendre des vues hétérodoxes devant l'Empereur, en soutenant que les dynasties anciennes de la Chine ne sont qu' «allégoriques » ${ }^{40}$. Dentrecolles critique également les méthodes de numérologie employées par Bouvet qu'il juge «arbitraires ${ }^{41}$.

37. ARSI, JS $168, \mathrm{f}^{\circ} 322 \mathrm{v}$.

38. ARSI, JS 174, 45-46; 51-54; 55-57; 65-70; 71-72; 93-95; 110-115. D'importants extraits ou des résumés de ces lettres sont donnés dans Thomaz de Bossierre 1982 : 14-16.

39. Dentrecolles à Contancin, Raozhou, 23 juillet 1711 (ARSI, JS 174, f ${ }^{\circ}$ 56; voir aussi Thomaz de Bossierre 1982: 15). «Ie defends in virtute s [anc] ta obedientia de proposer a l'Empr, comme chose seure, le terme de la durée du monde, le fixant v. g. au jour qui finiroit dix mille ans de cette durée, pretendant que l'autorité des Kims [Jing] est claire sur cela, et qu'elle est d'ailleurs irrefragable à raison de la révélation.»

40. Dentrecolles à anonyme, Raozhou, le 13 février 1713 (BnF, Ms NAF 6556, fo 85v-86): «On conclura que toutes l'ancienne cronique des livres classiques n'est point reelle mais purement allegorique, et que c'est un ouvrage qui contient toute autre chose que des faits reellement historiques. ie ne scai si $V^{e} R^{\text {ce }}$ voit tout le mal dont nous sommes menacés si l'Empr vient a s'appercevoir qu'on vise a cela [...] L'Empr s'en serait desia apperceu si un Chinois pouvoit s'imaginer qu'un European veut montrer que Yao, Xun [Shun], Vouen Vam [Wenwang], Vou Vam [Wuwang] et les autres grands roys ou ministres des trois fameuses dynasties de leurs livres classiques sont des personnes mythologiques. C'est ainsi que le P. Bouvet s'explique dans plusieurs de ses lettres...» De longs extraits de cette lettre sont donnés dans Thomaz de Bossierre 1982 : 17-21.

41. Dentrecolles à Bouvet, le 3 mai 1712, Raozhou (ARSI, JS 174, $\mathrm{f}^{\circ}$ 176-191). «Mon $\mathrm{R}^{\mathrm{d}}$ Pere, j'ai lu les escrits que V. R. a envoyé dans les provinces en differens temps [...]. Je ne suis pas versé dans les mathématiques, mais il me semble que dans la science des nombres tout va par demonstrations et que les principes de cette [?] science sont necessaires...» $\mathrm{F}^{\circ} 181$ : «[...] il me semble aussi sur la lecture de ses escrits, que les combinaisons qu'elle faict sont fort arbitraires, et qu'elle n'apporte point de raison et 
Mais on peut aussi sentir dans ces lettres l'opposition entre deux stratégies d'évangélisation, l'une «par le bas» et l'autre «par le haut», la première voulant «donner pour marque de notre mission que nous évangélisons les pauvres», chez lesquels Dentrecolles trouve «moins d'obstacles et plus de docilité aux vérités du salut que dans les grands et dans les puissants du siècle ${ }^{42}$ », la seconde procédant par l'initiation aux «mystères » qu'on pense découvrir dans les livres classiques, accessibles aux seuls érudits. La position de Dentrecolles sur ce point est sans équivoque:

Ce n'est pas le temps d'occuper les chinois a des questions trop relevées, [...] cela nuit à la simplicité et docilité d'esprit qui est la grande vertu des neophites et dans les infidelles une excellente disposition au don precieux de la foy ${ }^{43}$.

Cette position de Dentrecolles et la priorité qu'il donne aux couches inférieures de la société semblent trouver un écho dans la seule traduction que nous ayons de lui qui soit clairement datée de la période de son séjour au Jiangxi. La source principale traduite est le Fu hui quan shu de Huang Liuhong (1651-?), titre ainsi rendu par Dentrecolles: L'Art de rendre le peuple heureux ${ }^{44}$. Le motif de la traduction, passé sous silence lors de sa publication dans la Description, est explicite dans le manuscrit conservé à la $\mathrm{BnF}^{45}$ : en voyant fonder par des fonctionnaires locaux à Raozhou des «y hio» [yixue], écoles caritatives pour enfants pauvres, Dentrecolles a «eu la curiosité de s'instruire dans les livres». Mais contrairement à de nombreux récits sur la Chine de cette époque qui font l'éloge du système chinois, Dentrecolles cherche à montrer le «prodigieux avantage que nous avons en Europe» en matière d'éducation:

d'engagement selon la doctrine chinoise, pour chercher plutost ce nombre qu'un autre, pour le chercher plutost dans le lo chu (Luoshu) que dans le ho tou (Hetu), plutost par la multiplication que par l'addition; car i'ai omit dire qu'il n'y a point de nombre qu'on ne put trouver en combinant ceux de ces deux figures, [...] que les nombres des deux grandes periodes de V. R. se peuvent tirer en bien des manieres soit du ho tou soit du lo chu indifferemment...»

42. Propos rapportés dans la lettre de Foucquet au Duc de la Force, Nanchang, le 26 novembre 1702 (LEC: 57).

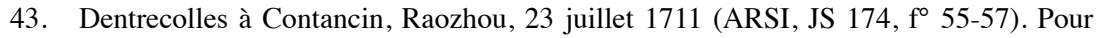
plus de détails concernant le débat entre jésuites français autour du figurisme, voir Wu 2013: 121-158.

44. Du Halde 1735 : 259. Isabelle Landry-Deron a identifié le $F u$ hui quan shu comme la source de cette traduction (Landry-Deron 2005). Il existe une traduction anglaise intégrale du Fu hui quan shu: Huang [1696] 1984.

45. BnF, MF 34566, fo $14 \mathrm{v}-27$. 
Les traductions de François-Xavier Dentrecolles, missionnaire en Chine

Si l'on peut un jour fonder icy un College, tel que nous en avons en France, les Chinois sentiront avec étonnement la supériorité de nos voiës Européanes sur celles de leurs Sages.

Il conclut par une demande adressée à son correspondant :

Je conjure votre Révérence de s'employer, pour faire fonder par les personnes dévotes, dans les endroits où nous avons des Églises, des écoles pour nos jeunes Chrétiens. C'est un préservatif bien nécessaire pour eux contre l'erreur et la corruption, dont leur esprit et leur cœur sont à cet âge si susceptibles entre les mains d'un Infidèle ${ }^{46}[\ldots]$.

Si Dentrecolles n'est pas le seul missionnaire à exprimer son souci d'apporter une éducation appropriée aux enfants des chrétiens, sa démarche consistant à s'informer des pratiques locales et à les présenter à l'aide d'une traduction semble unique. Que cherche-t-il dans cet ouvrage? Le Fu hui quan shu est l'un des plus célèbres manuels pour fonctionnaires (guanzhen) ${ }^{47}$, qui restera jusqu'à la fin des Qing un best-seller, disponible «partout dans les librairies ${ }^{48}$ », et que l'on trouve «entre les mains de presque chaque fonctionnaire débutant ${ }^{49} \gg$. L'existence de ces manuels se justifie par le décalage supposé entre deux registres de savoir, celui des lettrés et celui des administrateurs : les fonctionnaires débutants, frais émoulus des examens qui sélectionnent ceux qui connaissent le mieux les classiques, n'ont pas nécessairement les compétences techniques requises sur les lois, les institutions et la société qu'ils doivent gouverner ${ }^{50}$. On trouve dans le $F u$ hui quan shu - véritable encyclopédie sur la bureaucratie chinoise au niveau d'un district - des admonestations à la probité et à la prudence, une description sans concession de la misère et de la violence qui règnent dans la société rurale, des conseils pratiques pour accomplir son devoir de magistrat et, parfois, des projets contribuant au bien public. Ce sont bien les chapitres préconisant la création d'écoles caritatives pour pauvres - l'expression d'un idéal plus que d'une réalité - que traduit le jésuite.

46. BnF, MF $34566, \mathrm{f}^{\circ} 13$.

47. Sur le Fu hui quan shu, voir Will 1989 : 71-80. P.-E. Will prépare actuellement une bibliographie critique des guanzhen: Official Handbooks and Anthologies of Imperial China: A Descriptive and Critical Bibliography.

48. Selon Liu Heng (?-1841), qui a fait sa carrière dans le Sichuan. Will $2003: 33$.

49. Selon Zhou Shouchang (1814-1884), fonctionnaire à Pékin. Du 2011: 102.

50. Will $2003: 7-10$ et 28-36. 
Pour Dentrecolles, la lecture de ce manuel semble d'abord répondre à un besoin personnel de connaître les us et coutumes de la Chine. Les connaissances qu'il en tire se trouvent complétées par ses propres observations, démarche épistémologique qui se reflète dans une structure textuelle faisant alterner traduction et «remarques du traducteur». Après avoir traduit les propos de l'auteur du Fu hui quan shu qui enjoint, en référence à un idéal confucéen, les «Lettrez aisés» et les «gens riches qui en ont la charge» de «se faire un plaisir de s'unir», pour fonder des écoles qui «s'ouvrent principalement aux enfans du pauvre peuple qui, sans ce secours, ne sçauroient jamais avancer dans les Lettres ${ }^{51} »$, Dentrecolles remarque que, dans la réalité, les «y-hio [yixue] [...] sont rares en Chine, autant je puis le juger ${ }^{52} \gg$. D'après lui, les missionnaires auraient beaucoup à gagner s'ils pouvaient combler ce vide, en raison de l'importance du rapport entre maître-disciple dans la culture chinoise:

La qualité de maitre, ou Sien sem (xiansheng), ne se perd point à l'égard de ceux qui ont été disciples. Celui, dit le proverbe, qu'on a une fois reconnu pour maitre, doit être regardé durant toute la vie comme pere. C'est sans doute, selon ce principe Chinois, que le fameux Ministre d'Etat Paul Siu, grand Protecteur de nôtre sainte Religion ayant appris la mort du Missionnaire qui l'avoit instruit \& baptisé, prit le deüil, \& le fit prendre à toute sa famille, comme il avoit fait pour son propre pere ${ }^{53}$.

Ces informations tirées d'un manuel pour fonctionnaires ont sans doute aussi été rassemblées en vue d'un type d'intervention dans laquelle le missionnaire se reconnaît. Il est révélateur que Dentrecolles ait laissé une note sur le terme muzai, par lequel Huang Liuhong désigne le magistrat local: «Le mot Chinois est Moutsai, Mou, signifie Pasteur, Tsai, signifie Gouverneur ${ }^{54}$.» Il explique par ailleurs qu' «à la Chine le Mandarin de ville est le chef de police et de la religion $^{55} \gg$. Il y a effectivement superposition entre le projet missionnaire et les domaines d'intervention traditionnels du fonctionnaire - en l'occurrence l'éducation élémentaire - et analogie entre les valeurs confucéennes d'humanité (ren) et de justice (yi), piliers de l'éthique du fonctionnaire selon Huang Liuhong, et les vertus chrétiennes de «charité» et de «piété», termes utilisés par Dentrecolles dans sa traduction. Mais le jésuite dessine aussi une

51. Du Halde 1735: t. 2, 260.

52. Bnf, Ms Fr 17238, f 23 ; Du Halde 1735: t. 2, 263.

53. BnF, Ms Fr 17238, f ${ }^{\circ} 24 v$; Du Halde 1735 : t. 2, 264. Il s'agit de Xu Guangqi (15621633), baptisé en 1603 par João da Rocha (1565-1623).

54. Du Halde 1735 : t. 2, 263.

55. Dentrecolles à Madame *, Pékin, le 19 octobre 1720 (LEC : 302). 
hiérarchie entre la «vraie charité» chrétienne et la «compassion naturelle» des «gentils»: les écoles où l'on accueille gratuitement des enfants pauvres ne seront « véritablement des y hio, écoles de piété dans le motif, dans les moyens et dans les suites ${ }^{56} \gg$ que si l'on réussit à leur insuffler un contenu chrétien.

Nous ignorons les suites qu'a pu avoir ce projet comme, de manière plus générale, nous savons peu de chose sur l'œuvre éducative des missionnaires catholiques en Chine avant le XIX ${ }^{\mathrm{e}}$ siècle. En 1722 - bien avant la publication de cette traduction dans la Description en 1735 -, une lettre de Dentrecolles nous apprend toutefois que dans «une chrétienté qui est sur la route allant en Tartarie», il y avait «trois bandes de jeunes chrétiens au nombre de quarante, de divers ages, ayant trois maîtres d'escoles xns (chrétiens) pour les instruire, surtout dans la doctrine xne, dont ils respondoient à merveille, plusieurs sachant par cœur un assez gros cathechisme du P. Brancati ${ }^{57}{ }$.

\section{À Pékin, avant l'interdiction du christianisme (1720-1724)}

À la fin de l'année 1719, Dentrecolles est déchargé de sa fonction de supérieur de la mission française et part prendre la tête de la résidence de Pékin. Cette mutation ne semble au départ qu'une solution ad hoc pour répondre à une crise disciplinaire interne: il faut une figure d'autorité pour maîtriser les excès de tempérament de Foucquet qui refuse de reconnaître comme supérieur de résidence tous les candidats éventuels déjà sur place ${ }^{58}$. Dentrecolles accepte cette mutation à contrecœur: les missions de provinces manquent cruellement d'effectifs ${ }^{59}$, et il pressent des mesures anti-chrétiennes imminentes de la part de l'empereur, le pape ayant refusé tout compromis au sujet des rites chinois ${ }^{60}$. Mais Dentrecolles s'adapte vite à la réalité de la

56. BnF, MF 34566, f ${ }^{\circ} 13-14$.

57. Dentrecolles à Guibert, Pékin, le 12 octobre 1722 (ARSI, JS 199-1, f 306).

58. Dentrecolles à Guibert, Jiujiang, le 9 septembre 1718 (ARSI, JS 182, fo 30-38); Dentrecolles à Nyel, Jiujiang, le 18 septembre 1718 (JS 182, $\mathrm{f}^{\circ}$ 41-42).

59. Dentrecolles à Guibert, Jiujiang, le 9 septembre 1718 (ARSI, JS 182, fo 32): «[...] pouvoit ie quitter mes eglises, m'aller ietter a Pekim ou les embarras causés par la constitution estoient les plus grands, d'ailleurs j'eus une grosse maladie en juillet qui me laissa long temps convalescent, et durant la convalescence le P. de Chavagnac qui m'assistait fut attaqué et mourant entre mes bras me laissa chargé de 4 eglises outre les miennes propres : il fallû donc me determiner.»

60. Dentrecolles à Nyel, Jiujiang, le 30 septembre 1719 (ARSI, JS 182, fo 63): «[...] ie vous dirai en deux mots l'estat de la mission, elle est à demy perdue, les Xns n'approchant point des sacremens, et elle va achever de se perdre, si dans toute cette mousson il n'est arrivé en Chine aucun ordre de sa Sté, dont l'Empr soit content et qui 
capitale; dès octobre 1720, il envoie la traduction d'un second passage du Fu hui quan shu, exposant cette fois-ci des projets de secours aux pauvres, et notamment aux enfants abandonnés, dont le très grand nombre représente la misère en Chine d'une manière particulièrement choquante pour la sensibilité européenne ${ }^{61}$; de surcroît l'abandon des enfants semble sévir davantage à Pékin qu'à Raozhou ${ }^{62}$. Dentrecolles continue également à diriger à distance les missions du Jiangxi, et y retourne au moins une fois à la fin de l'année 1721, après que l'église de Jingdezhen a subi des dégâts lors d'une émeute dirigée contre les «musulmans ${ }^{63}$ : ce séjour d'un mois est l'occasion d'une seconde enquête sur la porcelaine, dont il rend compte depuis Jingdezhen dans une lettre datée de janvier $1722^{64}$. En septembre 1722, il espère quitter bientôt la capitale de façon définitive ${ }^{65}$; mais cela ne se fera pas. En janvier 1724, le couperet tombe : l'empereur Yongzheng (r. 1723-1735) interdit l'évangélisation et ordonne l'expulsion de tous les missionnaires des provinces. Dentrecolles restera à Pékin jusqu'à la fin de sa vie.

La plupart des écrits à caractère savant de Dentrecolles sont postérieurs à son arrivée à Pékin, ce qui peut s'expliquer par la plus grande sédentarité de sa vie pékinoise, qui offre un cadre propice au travail savant. À l'égard de ses

tirent les missionnaires d'embarras; i'ai crû le danger si present, que i'ai représenté à N R P Supr. pour différer de quelques mois mon voyage pour Pekim, iugeant que j'allais là pour en estre chassé, n'ayant aucune habileté pour le service de l'Empr [...].»

61. Mungello $2008: 7-8$.

62. Dentrecolles à Madame *, le 20 octobre 1720 (LEC : 295). La destinataire est une bienfaitrice anglaise, dont Dentrecolles dit de ne pas connaître l'identité, mais qui a financé le baptême des enfants abandonnés moribonds. Dentrecolles explique la différence de situation entre la capitale et une ville provinciale de second ordre dans une note de traducteur: «À Jao-tcheou [Raozhou], et dans les villes qui se trouvent aux environs, on expose peu d'enfans: les pauvres les mettent pendant la nuit à la porte de l'hôpital; en hiver il y a une espèce de crèche avec du coton, on y met l'enfant. Le portier accourt et prend l'enfant, qu'il remet entre les mains des nourrices. Je viens d'apprendre qu'il ne me sera pas difficile de gagner le portier de l'hôpital de Jaotcheou, et de procurer, par ce moyen-là, le baptême à plusieurs enfans moribonds.» ( $L E C:$ :296.) Sur la réception de cette lettre, voir R. Hsia $2006: 41$.

63. LEC : 308-309. Cette «émeute»n'est pas enregistrée dans les sources locales de Jingdezhen: je remercie le Dr. Liu Zhaohui (Université de Fudan) d'avoir confirmé leur silence à ce sujet.

64. Dentrecolles au Père ***, le 23 janvier 1722 (LEC : 309-316).

65. Dentrecolles à l'assistant de France Guibert, Pékin, le 22 septembre 1722: «[...] me voilà au bout de ma superiorité et par consequent prest à quitter Pekim.» (ARSI, JS 199, fo 297; voir aussi Thomaz de Bossierre 1982 : 57.) 
confrères figuristes qui revendiquent une expertise en lettres et philosophie chinoises, il ne se contente plus d'exercer une censure mais entend désormais les combattre avec leurs propres armes. Ainsi, en traduisant en 1722 un traité sur «la monnaie qui en différents tems a eu cours en Chine ${ }^{66} »$, un catalogue numismatique illustré intitulé Quan zhi (Mémoire sur les monnaies) de Hong Zun (1120-1174) ${ }^{67}$, Dentrecolles explique à sa hiérarchie romaine qu' «il y a des choses assez curieuses et utiles » dans ce livre qui prouvent que :

[...] les trois fameuses dynasties du Chu Kim [Shujing, Classique des Documents], c'est-à-dire celle des Hia, des Cham et des Tcheou ne sont point une mythologie, comme disent les PP. Bouvet et Foucquet, mais qu'elle ont esté tres réelles, puisqu' on trouve dans le tresor royal des pieces de monnaye de plusieurs Empereurs de ces anciennes dynasties ${ }^{68}[\ldots]$.

La différence essentielle entre Pékin et les provinces semble tenir plus encore à la facilité d'accès à l'actualité savante de l'Europe. Il est sans doute opportun de rappeler à cet égard que ce n'est qu'après le départ de Dentrecolles pour la mission en 1698 que les polémiques sur la Chine commencent à prendre de l'ampleur dans la sphère publique en Europe: la condamnation en Sorbonne des Nouveaux Mémoires sur l'état présent de la Chine (1696) du jésuite Louis Le Comte (1655-1728) date de 1700; la traduction française du Traité sur quelques points de la religion des Chinois de Niccolò Longobardo (1565-1655), successeur de Matteo Ricci, mais critique de la stratégie d'accommodation de celui-ci, est publiée en 1701. Le philosophe Nicolas Malebranche (16381715), puisant dans cette source et dans ses conversations en 1707 avec Artus de Lionne, des Missions étrangères de Paris (1655-1713), un détracteur des jésuites qui a lui-même travaillé en Chine, publie en 1708 un dialogue fictif intitulé Entretien d'un Philosophe Chrétien \& d'un Philosophe Chinois, dans lequel il compare la notion néo-confucéenne de $l i$ avec l'«athéisme» de Spinoza ${ }^{69}$. Dans la correspondance de Dentrecolles avec Rome et Paris, rien n'indique que sa «petite bibliothèque européenne ${ }^{70}$ » ait été actualisée pendant les vingt ans qu'il a passés au Jiangxi, où elle avait suscité la curiosité des lettrés. Les bibliothèques jésuites à Pékin sont plus riches et plus à jour. Elles possèdent surtout les principaux périodiques savants européens, comme

66. Du Halde 1735 : t. 2, 164-167.

67. Hong 1991.

68. Dentrecolles à Guibert, Pékin, le 12 octobre 1722 (ARSI, JS 199-1, f ${ }^{\circ}$ 305v.).

69. Mungello 1980; Landry-Deron $2002: 87-88$.

70. Du Halde 1735 : t. 2, 266. 
les Mémoires de Trévoux, l'Histoire de l'Académie royale des Sciences, les Philosophical Transactions de la Royal Society de Londres ou les Acta Eruditorum de Leipzig ${ }^{71}$. Invention de la seconde moitié du XVII ${ }^{\mathrm{e}}$ siècle ${ }^{72}$, les périodiques qui, à l'instar du périodique jésuite Mémoires de Trévoux veulent «donner des extraits de tous les livres de sciences imprimés en France, en Espagne, en Italie, en Allemagne et dans les royaumes du Nord, en Hollande, en Angleterre, etc. ${ }^{73}{ }^{\prime}$, constituent un nouvel outil de communication des savants européens. Si des ouvrages savants sont disponibles dans plusieurs résidences jésuites des provinces, seules celles de Pékin semblent avoir constitué des collections de périodiques ${ }^{74}$.

Grâce à ces ressources disponibles à la capitale, Dentrecolles semble s'être mis au fait des débats savants en Europe au cours des deux décennies qui venaient de s'écouler ${ }^{75}$. Dorénavant, ses traductions répondent à des polémiques européennes, et non plus à un besoin de connaissance du terrain chinois où il exerce son ministère. Il en va ainsi de deux traductions touchant à des questions doctrinales. Dans une note de traducteur de la première, intitulée Dialogue, où un Philosophe Chinois moderne nommé Tchin expose son sentiment sur l'origine \& l'état du Monde ${ }^{76}$, Dentrecolles attaque l'Entretien

71. Nous pouvons avoir une idée précise des périodiques que possède la bibliothèque des jésuites français de Pékin grâce à la correspondance d'Antoine Gaubil. Par exemple, Gaubil à Souciet, le 23 septembre 1732: «Des Mémoires de Trévoux 1701, 1702, 1703, 1704 nous manquent; juillet-août, 7bre [sic pour septembre] de 1706, les 9 derniers mois de 1720 , et les 9 premiers mois de 1726 nous manquent»; Gaubil à anonyme, probablement Souciet, Pékin, le 25 septembre 1732: «Des Leipsik, 1722 et 1724 manquent, et nous avons jusqu'à 1727 inclus» (Gaubil $1970: 333$, 338; voir aussi Golvers 2012, vol. $1:$ 170).

72. Peiffer et Vittu 2008.

73. Mémoires de Trévoux, 1701 : iii-iiij, «préface».

74. Joseph de Prémare est le seul jésuite qui dit avoir lu des périodiques hors de Pékin (à Jiujiang en 1723 et à Canton en 1725): il s'agit sans doute d'une exception qui confirme la règle, les deux villes étant des étapes obligées par lesquels les livres arrivés d'Europe sont acheminés jusqu'à Pékin (voir Golvers 2012: 475). Je remercie Noël Golvers pour son aide sur cette question.

75. Je parle ici des débats savants, par opposition aux disputes internes à l'Église. Bien entendu, la démarcation entre les deux n'est pas nette, la qualité d'homme d'Église et celle de savant étant souvent cumulées par la même personne. Mais la question de l'érection de la mission française en vice-province, par exemple, au sujet de laquelle Dentrecolles a échangé une correspondance interminable avec Rome durant toute l'année 1712, n'a suscité nul intérêt en dehors de la Compagnie de Jésus.

76. Du Halde 1735: t. 3, 42-64. Curieusement, l'original chinois est un roman vernaculaire, le Dou peng xian hua (Propos oisifs sous la tonnelle aux haricots). Voir Wu 2012 pour 
d'un philosophe chrétien \& d'un philosophe chinois de Malebranche, en citant textuellement le compte rendu hostile de ce dernier ouvrage publié en 1708 dans les Mémoires de Trévoux. Ce compte rendu lui-même citait quelques lignes de Malebranche:

$\mathrm{Au}$ reste le Philosophe Chinois, moins par le génie propre de sa nation, que par l'admiration de tant de subtilitez, ne se rend point contentieux. Il expose ainsi son systême: «Nous ne recevons que la Matiere, \& le $L y$, cette souveraine vérité, sagesse, justice, qui subsiste éternellement dans la Matière, qui la forme \& la range dans le bel ordre que nous voyons, \& qui éclaire aussi cette portion de matiere épurée \& organizée, dont nous sommes composez. Car c'est nécessairement dans cette souveraine Verité, à laquelle tous les hommes sont unis, les uns plus, les autres moins, qu'ils voyent les veritez \& les loix éternelles, qui font le lien de toutes les Societez ${ }^{77}$.»

Et Dentrecolles de reprendre dans sa note de traducteur du Dialogue:

[...] ce R. Pere (Malebranche) assûre que selon le systême de la Philosophie Chinoise, toutes les véritez sont vûës dans le $L i, \&$ c'est selon ce systême qu'il a imaginé dans l'Ouvrage intitulé, Entretien d'un Philosophe Chrétien avec un Philosophe Chinois: Nous ne recevons que la Matiere, \& le Ly, cette souveraine vérité, sagesse, justice, qui subsiste éternellement dans la Matière, qui la forme \& la range dans le bel ordre que nous voyons, \& qui éclaire aussi cette portion de matiere épurée \& organisée, dont nous sommes composez. Car c'est nécessairement dans cette souveraine Verité (le li), à laquelle tous les hommes sont unis, les uns plus, les autres moins, qu'ils voyent les veritez \& les loix éternelles, qui font le lien de toutes les Societez. [...] Malheureusement ce langage est nouveau \& inoüi à la Chine, \& il n'y a point de Lettré qui ne fût étrangement surpris d'apprendre qu'on lui fît tenir un pareil discours ${ }^{78}$.

L'identité au mot près des deux citations, ainsi que l'absence de source prouvant l'existence de l'ouvrage de Malebranche dans les bibliothèques jésuites à Pékin, laisse à penser que Dentrecolles s'engage dans le débat autour de cet ouvrage en s'appuyant uniquement sur l'information rapportée dans le périodique, sans avoir eu directement accès à l'ouvrage critiqué.

l'identification de l'original; et Wu 2013: 169-227 pour une analyse détaillée de la traduction.

77. Mémoires de Trévoux, juillet 1708: 1134-1143, décembre 1708: 1977-2003.

78. Du Halde 1735 : t. 3, 56. 
La seconde traduction, intitulée «Caractère et mœurs des chinois, par un Philosophe moderne de la Chine ${ }^{79} »$, est envoyée à Rome et à Paris. À ses supérieurs romains, Dentrecolles explique sobrement que dans cette «version d'un livre chinois ouvrage d'un philosophe chinois peu ancien, il y a de bons endroits, propres a iustifier nos anciennes pratiques». Dans sa lettre à Du Halde, en revanche, sa cible est désignée par une question rhétorique:

[...] on me demandera peut estre, que iugez vous de votre auteur, le faites vous deiste ou bien athée?

\section{Réponse:}

Ie dirai seulement sur ce point, que si mon escrit passoit en angleterre, et qu'il y fut examiné par Mr Hyde ou par ses vrais disciples, cet illustre auteur de l'ouvrage tant loüé historia religionis veterum persarum declareroit mon auteur chinois un deiste [...] mais si mon escrit tomboit entre les mains de Mr Tolandus, autre escrivain anglois, dont il est fait mention dans les memoires curieux de Lipsic [Leipzig] au tome de l'année 1709 et ailleurs, mon auteur chinois courreroit le risque a un tel tribunal d'estre iugé un vrai athée ${ }^{80}[\ldots]$.

C'est dans le numéro de septembre 1709 des Mémoires de Trévoux que paraît une critique de l'Adeisidaemon, sive Titus Livius a superstitione vindicatus... Annexae sunt ejusdem Origines judaicae de John Toland (1670-1722). Ce dernier, selon son critique, soutient que «la Nature est son Dieu et toutes les autres religions sont superstitions »; pour cela, il invoque non seulement des auteurs grecs et latins, mais aussi «l'autorité des Lettrez Chinois, \& des Philosophes d'Orient ${ }^{81}{ }^{1}$. Au Jiangxi comme à Pékin, Dentrecolles a toujours défendu avec ardeur et verve la position officielle des jésuites dans les débats internes de l'Église, contre les dissidents de la Compagnie comme contre l'hostilité des autres ordres; ce n'est néanmoins qu'avec la facilité nouvelle d'accès à l'actualité savante de l'Europe que lui procure son séjour à la capitale qu'il peut contribuer à la défense de la position jésuite dans la sphère publique européenne, où les philosophes sont en train de se saisir des sujets ayant trait à la Chine.

79. Du Halde 1735: t. 3, 131-185. (BnF, Ms. Fr.17238, f ${ }^{\circ}$ 111-174; ARSI, JS 156.)

80. Dentrecolles à Du Halde, Pékin, le 14 août 1722 (ARSI, JS 156, f ${ }^{\circ}$ D-E).

81. Mémoires de Trévoux, septembre 1709: 1604-1606. 
Les traductions de François-Xavier Dentrecolles, missionnaire en Chine

\section{À Pékin, après l'interdiction du christianisme (1725-1741)}

L'année 1726 marque un nouveau tournant dans la carrière de Dentrecolles. Il signe en cette année-là un long traité intitulé Réflexions pour l'intelligence d'un point important dans l'histoire de la Chine ${ }^{82}$, qui ne cite aucune source chinoise, mais fait étalage d'érudition sur l'histoire des débuts de la Chrétienté, époque où de nombreux compromis furent consentis à l'égard des pratiques païennes, pour appeler à des «permissions adoucissantes... pour rendre plus facile l'obéissance au dit precepte apostolique ${ }^{83} »$. C'est son dernier écrit portant sur l'entreprise missionnaire, le dernier envoyé à Rome aussi. Il tourne la page, et passe de l' «édifiant» au «curieux».

Ce déplacement de son champ d'intérêt est contemporain des efforts d'autres jésuites français de Pékin - notamment Antoine Gaubil (1689-1759) et Dominique Parrenin (1665-1741) - pour renouer des liens avec l'Académie des sciences de Paris ${ }^{84}$, sans que l'on puisse affirmer qu'il y ait eu concertation entre eux et Dentrecolles. Pour ce dernier, en tous cas, l'intérêt pour les «arts » chinois remonte à son séjour au Jiangxi, pendant lequel il a fait preuve de son talent d'observateur avec l'enquête sur la porcelaine de Jingdezhen. Sa correspondance de Pékin après 1726 s'inscrit dans un même schéma de pensée : il s'intéresse aux savoirs naturalistes et techniques qui présentent une utilité immédiate pour la France, qu'il s'agisse de la possibilité d'acclimatation d'une plante ou de l'amélioration d'un savoir-faire artisanal. Il écrit ici comme sujet du roi de France, au service de ses intérêts, dans un esprit de concurrence avec d'autres puissances maritimes. En filigrane de ses suggestions d'acclimatation notamment, on voit se dessiner l'espace colonial français - la Guyane, les Antilles, Madagascar, et «nos îles » en général - comme dans la conclusion de sa traduction de 1736 sur le camphre:

Ne pourroit-on pas se procurer à Canton un petit plant de l'arbre d'où l'on tire le camphre, où je crois qu'il n'auroit pas de peine à croître ? [...] M. Lemery dit qu'il vient de Hollande en France du camphre de la Chine: peut-être que les Hollandois ont trouvé dans leurs îles, ou qu'ils y ont transporté d'ailleurs des arbres de camphre, et qu'ils le vendent sous le nom de camphre de la Chine ${ }^{85}$.

82. Biblioteca centrale di Roma Vittorio Emanuele II (BVE), GES 1597

83. BVE, GES $1597, \mathrm{f}^{\circ} 1$.

84. F. Hsia 1999 : 125-126, 153-155; Anonyme, $1^{\text {er }}$ mai 1723 (LEC: 330-346).

85. Dentrecolles à Du Halde, Pékin, le 8 octobre 1736 (LEC: 724). 
L'installation à Pékin a néanmoins introduit des changements subtils, à commencer par la distance qui le sépare de certains objets de savoir. À Jingdezhen étaient réunies l'extraction des matières premières et la production; à la capitale, les fabricants de fleurs artificielles, dont Dentrecolles a décrit avec admiration en 1727 l'art d' «imiter si bien la Nature», utilisent le tongcao (Tetrapanax papyrifer (Hook.) K. Koch, aralie à papier de Chine), une plante qui croît dans le sud de la Chine mais «point dans cette province» (celle qui entoure Pékin). Dentrecolles n'a donc pu «l'examiner par [lui]-même», et ce qu'il a «appris de ceux qui travaillent aux fleurs ne suffisoit pas» pour lui «donner des indices capables de [la] déterrer en France ${ }^{86}$ ». C'est ici qu'interviennent les sources écrites: après s'être informé du nom chinois de la plante (tongcao), il consulte un «herbier chinois» et traduit en détail les vertus médicinales et les caractéristiques de l'habitat naturel de celle-ci:

Si ces connoissances peuvent aider à trouver en Europe un arbrisseau semblable à celui qui fournit aux Chinois la matière dont ils font leurs fleurs artificielles, il ne sera pas difficile aux ouvriers européens d'imiter, et même de surpasser l'adresse chinoise dans cette sorte de travail ${ }^{87}[\ldots]$.

Mais plutôt que lui faire découvrir des savoirs radicalement nouveaux, le passage du Jiangxi à Pékin invite parfois le jésuite à revoir certains jugements qu'il avait portés sur des savoirs chinois observés. C'est le cas pour la variolisation, sujet d'une lettre en 1726 - sa seconde contribution savante la plus connue après la porcelaine.

En lisant depuis peu les mémoires de Trévoux de l'année 1724, je suis tombé sur l'extrait d'une lettre de M. de la Coste, dans laquelle il parle de l'insertion ou inoculation de la petite vérole; et je me suis souvenu d'avoir lu quelque chose d'approchant dans un livre chinois ${ }^{88}[\ldots]$.

L'extrait en question, paru en juin 1724 dans les Mémoires de Trévoux, est le premier compte rendu donné en France de la pratique de variolisation l'inoculation volontaire de la variole à des fins immunitaires - que Lady Montagu, épouse de l'ambassadeur anglais à Istanbul, venait d'introduire en Angleterre en $1721^{89}$. Les Mémoires de Trévoux plaident ardemment

86. Dentrecolles à Du Halde, Pékin, le 7 juillet 1727 (LEC: 547).

87. LEC: 546.

88. Dentrecolles à Du Halde, Pékin, le 12 mai 1726 (LEC: 535).

89. Mémoires de Trévoux, juin 1724: 1073-1090. 
pour qu'une expérience semblable soit menée en France - prise de position audacieuse de la part d'un journal jésuite, étant donné les débats intenses engageant physiciens, philosophes et théologiens ${ }^{90}$. Dentrecolles avait en réalité non seulement «lu quelque chose d'approchant», mais observé directement la variolisation lorsqu'il était dans le Jiangxi, où cette pratique était particulièrement courante ${ }^{91}$. Il l'a même rapportée en 1715 , dans une lettre consacrée au «progrès des travaux apostoliques» publiée dans les $L E C$. Après avoir raconté le baptême in extremis d'une fille morte de la variole, il décrit «la manière dont quelques médecins chinois traitent ceux qui ont la petite vérole». Il donne là une première description de la variolisation sommaire mais exacte, contenant des éléments clés comme l'introduction de l'inoculum par voie nasale, particularité de la technique chinoise. La pratique telle qu'il l'a observée était associée à une «superstition», le culte de la «déesse de la petite vérole », à qui le père de la défunte est allé rendre hommage ${ }^{92}$. Dentrecolles trouve la pratique extraordinaire, mais il «ajoute peu de foi à ce remède», et lui préfère «sans difficulté une prise de poudre de vipère ${ }^{93}$ ».

Pékin, capitale de la dynastie mandchoue, était en ce début du XVIII ${ }^{\mathrm{e}}$ siècle porteuse d'une histoire bien différente en la matière. Dentrecolles note très justement en 1726 que les Mandchous sont particulièrement vulnérables à cette maladie. L'empereur Shunzhi (r. 1644-1661) en était mort; Kangxi, qui y a survécu dans son enfance et qui en est resté marqué, fait inoculer ses enfants comme ses troupes ${ }^{94}$. D'après Dentrecolles, en 1724, une nouvelle campagne de variolisation est lancée par Yongzheng, qui «envoya [...] des médecins du palais en Tartarie, pour y mettre cette méthode en pratique ${ }^{95}[\ldots] »$. Dans ce nouveau contexte pékinois, la variolisation perd sa connotation superstitieuse et se rationalise comme pratique purement médicale ${ }^{96}$.

90. Sur le contexte européen, voir Puente Ballesteros 2006 : 90-91.

91. Selon le Dou zheng jin jing lu (1727) de Yu Tianchi, «la pratique de l'inoculation de la petite vérole débute sous le règne de Longqing (1567-1572) dans la préfecture de Ningguo (dans l'actuel Anhui, limitrophe à Raozhou)» (cité dans Needham, Lu et Sivin 2000 : 134). La fonction d'inoculateur semble être monopolisée par les médecins originaires de Ningguo, appelés les «maîtres de Ningguo» (Ningguo xiansheng) (Wang 2002 : 258).

92. Il s'agit du culte du Dou shen, ou Tian hua niang niang, extrêmement répandu dans le sud de la Chine. (Chang 1996 : 106-121; Wang 2002 : 265-269.)

93. LEC: 244-245.

94. Needham, Lu et Sivin $2000: 139-140$; Chang 2002.

95. LEC: 537.

96. Dentrecolles ne semble pas être au courant de l'appropriation du culte de la déesse de la variole par les Mandchous. (Voir Chang 2002 : 185.) 
Ce sont ces nouvelles connaissances autorisées par les milieux savants des diverses capitales - Pékin, Londres, Paris, Istanbul - qui ont remis en question le préjugé de Dentrecolles sur cette pratique qu'il avait observée dans les provinces. Toutefois, il s'abstient de prendre position, et veut «indifféremment citer les auteurs qui la décrient, et ceux qui la défendent» : il traduit un auteur moralisateur qui «vivoit à la fin des Ming», hostile à la variolisation, qui soutient que «le moyen le plus sûr de conserver [les enfants de la variole], c'est le soin qu'ont les parens de pratiquer constamment la vertu ${ }^{97} \gg$; il traduit aussi trois recettes qu'il a obtenues des «médecins au service du palais» subalternes, en leur offrant des «présents» et en leur promettant de ne pas diffuser en Chine «ce qu'on ne [lui apprend] qu'en faveur de l'Europe». Il est intéressant de noter au passage que le secret qui entoure certains savoirs et entrave leur circulation locale peut être levé en faveur d'un public assez distant pour ne pas entrer en concurrence avec les détenteurs de ces savoirs. Dentrecolles annote minutieusement ces recettes, faisant la synthèse de leurs points communs et montrant ceux sur lesquels elles divergent, et cherche dans $l^{\prime}$ «herbier [chinois]» des éclaircissements sur les plantes utilisées ${ }^{98}$. Il cherche également dans les sources chinoises des réponses aux questions soulevées par le débat en Europe: la cause de la variole, son universalité ou non dans le monde antique, l'origine de la pratique de variolisation, et si la «petite vérole artificielle est la même espèce que la naturelle ${ }^{99}{ }^{»}$. Il termine sur cette proposition, qui évoque une République des sciences élargie:

La connoissance des maladies et des remèdes n'a pas été si négligée à la Chine qu'on se l'imagine peut-être en Europe [...] le peu de ces livres que j'ai eu l'occasion de feuilleter m'ont persuadé que s'ils étoient traduits en notre langue, les médecins européens seroient contens de ce qu'ils disent ${ }^{100}[\ldots]$.

\section{Les limites de la circulation des livres}

Le parcours personnel de Dentrecolles, de France en Chine, du Fujian et du Jiangxi à Pékin, est entièrement dicté par une logique missionnaire, et non par des décisions de la cour impériale, comme ce fut le cas pour ses confrères qui y travaillaient. Il a cependant été l'un des contributeurs majeurs à la connaissance européenne de la Chine, y compris dans des domaines techniques spécialisés.

97. LEC: 536.

98. LEC : 537.

99. LEC: 540.

100. LEC: 543. 
L'accès aux livres imprimés, tant européens que chinois, a été crucial dans la formation de son expertise: c'est par ce biais qu'il identifie les débats européens et parvient à une juste compréhension des savoirs chinois. L'une des questions qui se posent lorsqu'on confronte l'évolution de l'œuvre écrite de Dentrecolles à son itinéraire géographique est la suivante: que peut nous révéler ce cas particulier quant à la distribution et la circulation des savoirs sous forme livresque en Chine à cette époque ?

La première conclusion que l'on peut tirer de notre analyse concerne la circulation des livres européens en Chine. En ce début du XvIII ${ }^{\mathrm{e}}$ siècle, cette circulation semble être marquée - beaucoup plus qu'à la fin des Ming - par la centralité de Pékin. La capitale impériale est le lieu de l'activité savante la plus intense des jésuites et attire par conséquent la plupart des ressources savantes en provenance d'Europe; elle tire ainsi avantage d'un investissement lourd dont les postes de mission dans le Jiangxi, consacrés exclusivement à l'évangélisation, ne semblent pas avoir bénéficié ${ }^{101}$. La différence entre la capitale et les missions des provinces tient ici à la disponibilité inégale des ressources intellectuelles.

En revanche, la géographie des livres chinois dont disposent les jésuites est beaucoup moins évidente à dessiner. Aucune politique bibliothécaire ne nous est connue en la matière, et les rapports que Dentrecolles entretient avec les livres chinois, à en juger par nos sources, semblent constants. Le monde des livres en Chine au début du XVIII ${ }^{\mathrm{e}}$ siècle est caractérisé par l'abondance et la circulation, et ce tant par le biais des manuscrits, dont l'importance persiste, que par celui des imprimés; pour comprendre ce monde, il faut prendre en compte à la fois les éditeurs commerciaux en plein essor depuis le $\mathrm{XVI}^{\mathrm{e}}$ siècle et les initiatives d'édition des autorités publiques à divers échelons ${ }^{102}$. Les livres chinois auxquels Dentrecolles a eu accès, dont le présent article ne peut fournir une analyse complète, s'inscrivent de plain-pied dans ce contexte: on y retrouve à la fois des imprimés commerciaux dont la diffusion est attestée à l'échelle de l'empire, des livres à vocation locale (comme la monographie locale de Fuliang), et des recettes médicales manuscrites obtenues par des contacts privés.

101. Sur la distribution des livres jésuites à l'intérieur de la Chine, voir Golvers 2012: 464478. Plus spécifiquement, sur les collections des bibliothèques jésuites à Pékin et en province, voir Golvers 2013.

102. Sur la coexistence d'imprimés et de manuscrits parmi les textes en circulation, voir McDermott 2006 : 44-79; sur les éditeurs commerciaux au début des Qing et le périmètre de diffusion géographique des textes imprimés, voir Brokaw 2007: 546-559. 
Cependant, l'accès aux connaissances par les livres avait ses limites. De manière révélatrice, Dentrecolles se plaint de l'impossibilité de trouver des écrits sur les procédés techniques de la porcelaine: "C'est pour des Chinois que se font ces recueils, et non pas pour les Européens; et les Chinois ne s'embarrassent guère de ces sortes de connoissances ${ }^{103}$.» L'aspect technique de sa lettre est ainsi entièrement basé sur ses enquêtes in situ et ses discussions avec les praticiens. Or, une description de la fabrication de la porcelaine existait bel et bien à cette époque dans un ouvrage imprimé: le Tian gong kai wu (Exploitation des Euvres du Ciel) de Song Yingxing (1587-1666?), auteur que Joseph Needham considérait comme «le Diderot de la Chine ${ }^{104} »$. Le fait que Dentrecolles, dont la lettre sur la porcelaine sera d'ailleurs reproduite in extenso dans l'Encyclopédie de Diderot et d'Alembert, se plaigne de l'absence de textes sur les techniques de la porcelaine laisse penser qu'il n'a jamais eu entre les mains l'ouvrage de Song Yingxing ${ }^{105}$. Cela n'est pas en soi surprenant: la célébrité du Tian gong kai wu, dont la diffusion à l'époque était certainement très limitée ${ }^{106}$, tient beaucoup à une construction historiographique a posteriori. Il est néanmoins frappant que d'un point de vue géographique, à intervalle de moins d'un siècle, Dentrecolles ait presque marché dans les pas de Song Yingxing, au sens le plus littéral du terme: Raozhou est situé sur la rive est du Lac Poyang, alors que Fengxin, la ville natale de Song Yingxing, se trouve à l'ouest de ce même lac. La première édition du Tian gong kai wu avait été imprimée à Nanchang, où le jésuite se rendait régulièrement. De plus, à l'époque même où Dentrecolles rédigeait depuis Pékin sa nouvelle série de lettres sur les «arts» des Chinois (1726-

103. Dentrecolles à Orry, Raozhou, le $1^{\text {er }}$ septembre 1712 (LEC: 207).

104. Needham et Wang Ling $1956: 13$.

105. Outre que Dentrecolles se plaint de l'absence d'écrits concernant la porcelaine, il y a des citations d' «auteur chinois» dans ses écrits qui contredisent les propositions du Tian gong kai wu; cela souligne l'originalité de la pensée de Song Yingxing, tout en confortant l'hypothèse que Dentrecolles n'a pas eu accès à son ouvrage. Par exemple, dans le chapitre sur le papier, Dentrecolles cite un «auteur chinois » non identifié qui affirme que les termes jian et ce font référence à des tablettes de bois et de bambou (Du Halde 1735: t. 2, 240), idée que réfute (à tort) Song Yingxing (Song Yingxing [1637] 1992 : 291); une lettre de Dentrecolles en 1734 contient une très longue argumentation en faveur de l'idée que les poursalanes (machixian) contiennent du mercure (Dentrecolles à Du Halde, Pékin, le 4 novembre 1734, LEC: 692-693), idée que Song Yingxing juge «sans fondement et téméraire» (wuduan kuangwang) (Song Yingxing [1637] 1992: 295).

106. Schäfer 2011 : 259. La première gravure du Tian gong kai wu n'aurait donné lieu qu'à une cinquantaine d'exemplaires sur papier. 
1737), non loin de la résidence jésuite, un exemplaire du Tian gong kai wu se trouvait dans la collection des livres du Palais et l'ouvrage était en cours d'intégration dans deux compilations impériales importantes: le Gu jin tu shu ji cheng [Collection d'écrits et d'illustrations d'antan et d'aujourd'hui] (1725), et le Qin ding shou shi tong kao [Compendium impérial sur les jours et les travaux] (1742) ${ }^{107}$. Ainsi la proximité physique n'est-elle pas une condition suffisante pour qu'un savoir soit disponible, même lorsque celui-ci ne fait pas l'objet d'un secret.

La mise en regard de l'œuvre savante de Dentrecolles et de son itinéraire dans l'empire chinois suggère qu'il existe certaines corrélations entre les sujets qui l'occupent et les lieux où il se trouve à chaque moment de sa carrière missionnaire. Mais chacune de ces séries d'éléments comporte de multiples dimensions. D'un côté, son travail de traduction, travail textuel par excellence, fait largement appel à des savoirs oraux et empiriques. De l'autre, le réseau missionnaire, qui détermine l'itinéraire de Dentrecolles, possède ses ressources et préoccupations propres qui ne recouvrent ni celles des milieux savants chinois ni celles de la cour impériale. La transmission d'un savoir de la Chine vers l'Europe est ainsi soumise à de multiples facteurs géographiques, sociaux, institutionnels. L'apparente systématicité du projet, qui aboutit à la publication de la Description de la Chine en 1735, ne saurait masquer la contingence à laquelle reste soumise cette transmission.

\section{BIBLIOGRAPHIE}

Aimé-Martin M. L. (éd.) ([édition originale 1702-1776, 34 vol.] rééd. 1853). Lettres édifiantes et curieuses concernant l'Asie, l'Afrique et l'Amérique, Tome Troisième: Chine. Paris, Société du Panthéon littéraire.

Brokaw Cynthia (2007). Commerce in Culture: The Sibao Book Trade in The Qing and Republican Periods. Harvard University Asia Center.

CAms Mario (2012). «Early Qing Geographical Surveys (1708-1716) as a Case of Collaboration between the Jesuits and the Kangxi Court». Sino-Western Cultural Relations Journal, vol. XXXIV: 1-20.

Chang Chia-feng (1996). «Aspects of Smallpox and its Significance in Chinese History», thèse de doctorat. School of Oriental and African Studies, University of London.

107. Schäfer $2011:$ 262-265. 
Chang Chia-feng (2002). «Disease and Its Impact on Politics, Diplomacy, and the Military: The Case of Smallpox and the Manchus (1613-1795)». Journal of the History of Medicine and Allied Sciences, ${ }^{\circ}$ 57.2: 177-197.

Collani Claudia von (1985). P. Joachim Bouvet S. J.: Sein Leben und sein Werk. Nettetal, Steyler Verlag.

Collani Claudia von (2007). «The First Encounter of the West with the Yijing ». Monumenta Serica, vol. LV : 227-387.

Du Halde Jean-Baptiste (comp.) (1735). Description géographique, historique, chronologique, politique et physique de l'Empire de la Chine et de la Tartarie chinoise, 4 vol., Paris, Le Mercier.

Du Jin 杜金 (2011). «Mingqing minjian shangye wenhua yunzuo xia de guanzhenshu chuanbo - yi fangke he shusi wei shijiao» 明清民間商業文化運作下的官筬 書傳播——以坊刻和書肆為視角. Fazhi yu shehui fazhan 法制與社會發展, $\mathrm{n}^{\circ} 99.3$ : 92-104.

Froger François (1926). Relation du premier voyage des François à la Chine, fait en 1698, 1699 et 1700 sur le vaisseau «l'Amphitrite », texte établi par E. A. Voretzsch. Leipzig, Verlag der Asia Major.

GaubIL Antoine (1970). Correspondance de Pékin.1722-1759, texte établi par Renée Simon. Genève, Librairie Droz.

Golvers Noël (2012). Libraries of Western Learning for China, Circulation of Western Books between Europe and China in the Jesuit Mission (ca 1650-ca 1750), vol. 1 : Logistics of book acquisition and circulation. Louvain, Ferdinand Verbiest Institute.

Golvers Noël (2013). Libraries of Western Learning for China, Circulation of Western Books between Europe and China in the Jesuit Mission (ca 1650-ca 1750), vol. 2 : Formation of Jesuit libraries. Louvain, Ferdinand Verbiest Institute.

Ho Ping-ti (1964). The Ladder of Success in Imperial China : Aspects of Social Mobility, 1368-1911. New York, Columbia University Press.

Hong Zun 洪遵 (rééd. 1996). Quan zhi 泉志, Shanghai, Shanghai guji chubanshe, Xuxiu siku quanshu 續修四庫全書, vol. 1112: 191-267.

HuANG Zongxi 黃宗羲 ([1676] rééd. 1985), Ming ru xue an 明儒學案, texte établi par Shen Zhiying 沈芝盈. Pékin, Zhonghua shuju.

Hsia Florence C. (1999). «French Jesuits and the Mission to China: Science, Religion, History », thèse de doctorat. Chicago, University of Chicago, Dept. of History.

Hsia Ronnie Po-Chia (2006). Noble Patronage and Jesuit Missions : Maria Theresia Von Fugger-Wellenburg (1690-1762) and Jesuit Missionaries in China and Vietnam. Rome, Institutum Historicum Societatis Iesu.

Huang Liuhong [éd. originale 1696], Duang Chu (trad.) (1984). A Complete Book Concerning Happiness and Benevolence: A Manual for Local Magistrates in Seventeenth-Century China. Tucson, University of Arizona Press.

Journal de Trévoux, ou Mémoires pour servir à l'histoire des sciences \& des beaux-arts (repr. 1968). Genève, Slatkine Reprints, t. 1, 8, 9 et 24 pour les années 1701, 1708, 1709 et 1724 . 
Les traductions de François-Xavier Dentrecolles, missionnaire en Chine

LANDRY-DERON Isabelle (2001). «Les mathématiciens envoyés en Chine par Louis XIV en $1685 »$. Archive for History of Exact Sciences, vol. 55: 423-463.

LANDRY-Deron Isabelle (2002). La Preuve par la Chine : la «Description» de J.-B. Du Halde, jésuite, 1735. Paris, Éditions de l'École des Hautes Études en Sciences sociales.

LANDRY-Deron Isabelle (2005). «Le Parfait Bonheur des Peuples. Traduction d'extraits d'un manuel chinois pour fonctionnaires de la fin du XVII ${ }^{\mathrm{e}}$ siècle». In BacquéGrammont, Jean-Louis, Pino, Angel et Khoury, Samaha (dir.), D’un Orient l'autre, Actes des troisièmes journées de l'Orient, Bordeaux, 2-4 octobre 2002. Paris/Louvain, Éditions Peeters : 109-121.

LEC. Voir Aimé-Martin, M. L. (éd.) (rééd. 1853).

McDermott Joseph P. (2006). A social History of the Chinese Book: Book and Literati Culture in Late Imperial China. Hong Kong, Hong Kong University Press.

Mémoires de Trévoux. Voir Journal de Trévoux (repr. 1968).

Menegon Eugenio (2009). Ancestors, Virgins and Friars: Christianity as a Local Religion in Late Imperial China. Cambridge, Harvard University Asia Center for the Harvard-Yenching Institute.

Mungello David E. (1980). «Malebranche and Chinese Philosophy». Journal of the History of Ideas, vol. 41, $\mathrm{n}^{\circ} 4: 551-578$.

Mungello David E. (2008). Drowning Girls in China: Female Infanticide in China since 1650.Lanham/Boulder/New York/Toronto/Plymouth, Rowman \& Littlefield Publishers.

Mungello David E. (1989). Curious Land: Jesuit Accommodation and the Origins of Sinology. Honululu, University of Hawaii Press.

NAQuin Susan et RawsKi Evelyn (1989). Chinese Society in the Eighteenth Century. New Haven, Yale University Press.

Needham Joseph, Lu Gwei-Djen et Sivin Nathan (2000). Science and Civilisation in China, vol. 6: Biology and Biological Technology, part. 6: Medicine. Cambridge, Cambridge University Press.

Needham Joseph et Wang Ling (1956). Science and Civilisation in China, vol. 1: Introductory Orientations. Cambridge, Cambridge University Press.

Ortroy Francis van (1910). «St. John Francis Regis». The Catholic Encyclopedia, vol. 8. New York: Robert Appleton Company. http://www.newadvent.org/ cathen/08464a.htm consultée le 13 août 2013.

Peiffer Jeanne et Vittu Jean-Pierre (2008). «Les journaux savants, formes de la communication et agents de la construction des savoirs $\left(\mathrm{XVII}^{\mathrm{e}}-\mathrm{XVIII}^{\mathrm{e}}\right.$ siècles $) »$. Dixhuitième siècle, vol. 40, $\mathrm{n}^{\circ} 1: 281-300$.

Pelliot Paul (1930). L'Origine des relations de la France avec la Chine: le premier voyage de «l'Amphitrite» en Chine. Paris, Librairie orientaliste Paul Geuthner.

Puente Ballesteros Beatriz (2006). «F.-X. d'Entrecolles S. J. and Chinese medicine: A Jesuit's Insights in the French Controversy Surrounding Smallpox Inoculation ». Revista de cultura International, $\mathrm{n}^{\circ}$ 16: 89-98.

SCHÄFER Dagmar (2011). The Crafting of the 10,000 Things : Knowledge and Technology in Seventeenth-Century China, Chicago, University of Chicago Press. 


\section{Wu Huiyi}

Sommervogel Carlos (1960). Bibliothèque de la Compagnie de Jésus. Héverlé-Louvain, Édition de la Bibliothèque S.J., Collège Philosophique et Théologique.

SoNG Yingxing 宋應星 ([1637] rééd. 1992). Tian gong kai wu 天工開物, texte établi et traduit en chinois moderne par PAN Jixing 潘吉星. Shanghai, Shanghai guji chubanshe.

StANDAERT Nicolas (éd.) (2001). Handbook of Christianity in China, 635-1800, vol. 1. Leyde, Brill.

Standaert Nicolas (2012). Chinese Voices in the Rites Controversy: Travelling Books, Community Networks, Intercultural Arguments. Rome, Institutum Historicum Societatis Iesu.

Thomaz de Bossierre Mme Yves de (1982). François Xavier Dentrecolles (Yin HongSiu Ki-Tsong) et l'apport de la Chine à l'Europe du XvIIIe siècle. Paris, Les Belles lettres.

Thomaz de Bossierre, Mme Yves de (1994). Jean-François Gerbillon, S. J. (16541707): mathématicien de Louis XIV, premier supérieur général de la Mission française de Chine. Louvain, Ferdinand Verbiest Foundation, K.U. Leuven.

WANG Qianjin 汪前進 (1995). «Huang yu quan lan tu cehui» 皇與全覽圖測繪. In Dong Guangbi 董光璧 (dir.), Zhongguo jinxiandai kexue jishu shi 中國近現代科 學技術史. Changsha, Hunan jiaoyu chubanshe: 131-174.

WANG Zhenzhong 王振忠 (2002). «Huizhou wenshu suojian zhongdou jiqi xiangguan xisu» 徽州文書所見種痘及其相關習俗. In Id., Huizhou shehui wenhuashi tanwei 徽州社會文化史探微. Shanghai, Shanghai shehui kexue chubanshe: 253-296.

WILl Pierre-Étienne (1989). «Bureaucratie officielle et bureaucratie réelle : sur quelques dilemmes de l'administration impériale à l'époque des Qing ». Études chinoises, vol. VIII, $n^{\circ} 1$ : 69-142.

WILl Pierre-Étienne (2003). «L'apprentissage du métier de fonctionnaire à la fin de la période impériale». In Nguyen Tri, Christine \& Despeux, Catherine (éd.), Éducation et instruction en Chine, vol. 2, «Éducation spécialisée». Paris et Louvain, Éditions Peeters: 7-47.

Witek John W. (1982). Controversial Ideas in China and in Europe: A Biography of Jean-François Foucquet, S. J. (1665-1741). Rome, Institutum Historicum S.I.

Wu Huiyi (2012). «Nouvelle identification d'une traduction chinois-français (1735)», Carnets du Centre Chine. Mis en ligne le 12 juillet 2012. http://cecmc.hypotheses. org/7299.

Wu Huiyi (2013). «Traduire la Chine au XVIII ${ }^{\mathrm{e}}$ siècle: les jésuites français traducteurs de textes chinois et la reconfiguration des connaissances européennes sur la Chine (1687-ca. 1740) », thèse de doctorat. Paris/Florence, Université Paris DiderotParis 7/Istituto Italiano di Scienze Umane.

Wu Min 吳旻 et HAN Qi 韓琦 (2004). «Liyi zhizheng he Zhongguo tianzhu jiaotu - yi Fuzhou jiaotu he Yan Dang de chongtu wei li»禮儀之爭和中國天主教徒-以福 州教徒和顏鐺的衝突為例. In Lishi yanjiu 歷史研究 $n^{\circ}$ 6: 83-91. 


\section{GLOSSAIRE}

$c e$ 策

Daoguang 道光

Dou peng xian hua 豆棚閒話

Dou shen 痘神

Dou zheng jin jing lu 痘疹金鏡錄

Fengxin 奉新

Fu hui quan shu 福惠全書

Fuliang xianzhi 浮梁县志

Fujian 福建

Fuzhou 福州, Fujian

Fuzhou 撫州, Jiangxi

Gu jin tu shu ji cheng 古今圖書集成

guanzhen 官筬

Hetu 河圖

Hong Zun 洪遵

Huang Liuhong 黃六鴻

Huang Zongxi 黄宗羲

jian 簡

Jiangnan 江南

Jiangxi 江西

Jiangyou Wang men 江右王門

jinshi 進士

Jingdezhen 景德鎮

Jiujiang 九江

Kangxi 康熙

li 理

Luoshu 洛書

machixian 馬齒莧

Ming ru xue an 明儒學案

Nanchang 南昌

Ningguo xiansheng 寧國先生

Qin ding shou shi tong kao 欽定授時通考

Quan zhi 泉志

Raozhou 饒州

ren 仁

Shujing 書經

Song Yingxing 宋應星

Tian gong kai wu 天工開物 
Wu Huiyi

Tian hua niang niang 天花娘娘

Tian xue ben yi 天學本義

tongcao 通草

xiansheng 先生

Yijing 易經

$y i$ 義

yixue 義學

Yu Tianchi 俞天池 